C-DEBI NextGen 2019: Early Career perspective on 'What's Next?' Upcoming Challenges and Opportunities

\title{
Primary authors:
}

Jacqueline Goordial ${ }^{1}$, Sarah K. Hu², Benjamin J. Tully ${ }^{3}$

Institutions and contact information

1, University of Guelph, goordial@uoguelph.ca

2, Woods Hole Oceanographic Institute, sarah.hu@whoi.edu

3, University of Southern California, tully.bj@gmail.com

Workshop attendees and co-authors: Rika Anderson, Anne Booker, James Bradley, Jesse Colangelo-Lillis, Kelle Frelle, Melody Lindsay, Olivia Nigro, Gustavo Ramirez, Brandi Kiel Reese, Lauren Seyler, Amy Smith, Andrew Steen, Elizabeth Trembath-Reichert, Tess Weathers

C-DEBI leadership sponsors: Jan Amend, Julie Huber, C. Geoff Wheat C-DEBI ExCom leadership: Steven D'Hondt, Andrew Fisher, John Heidelberg, Steven Finkel, Victoria Orphan, Beth Orcutt, Alfred Spormann

C-DEBI Administration: Rosalynn Sylvan, Nerissa Rivera-Laux

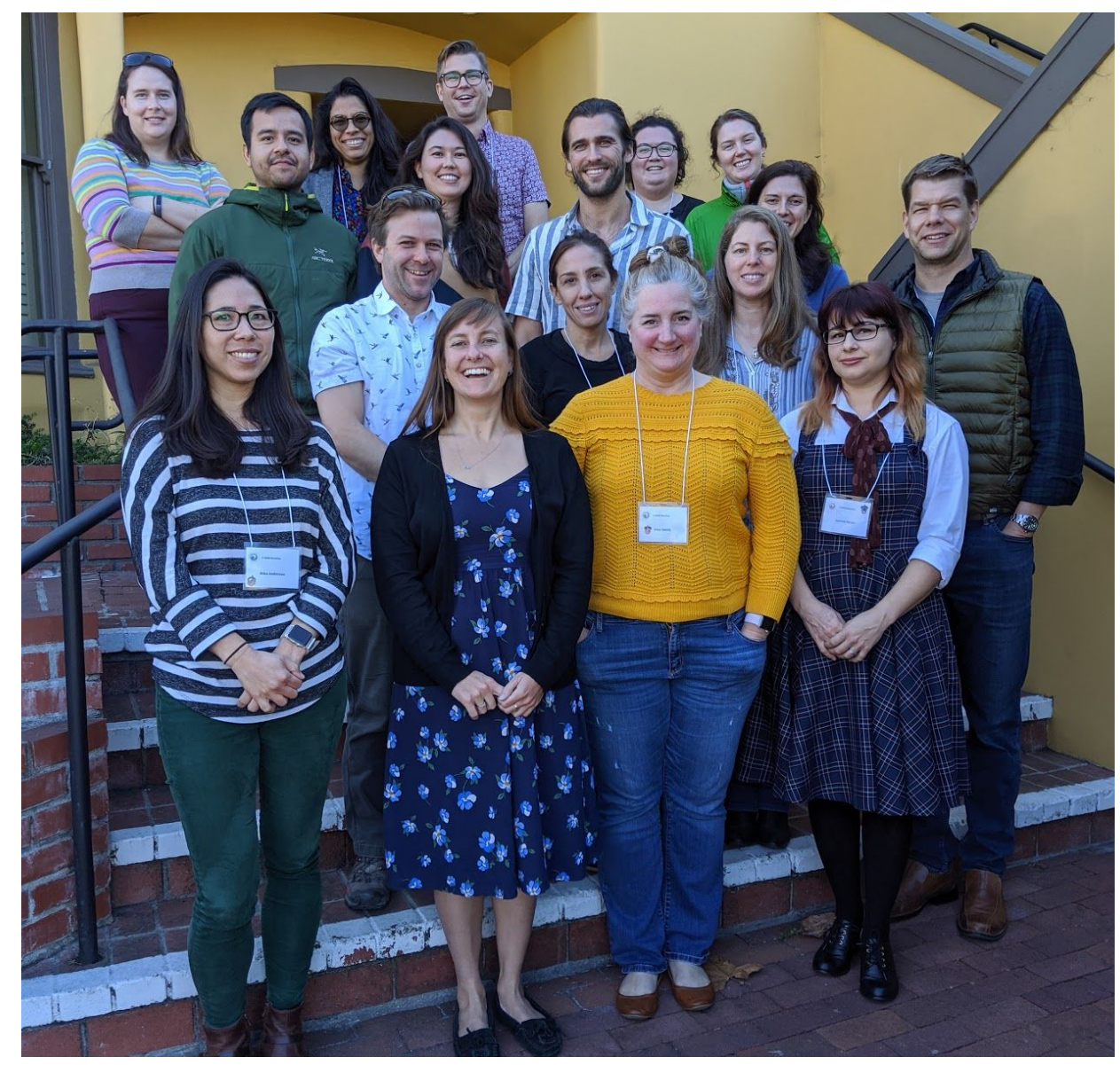




\section{Introduction}

The deep biosphere (subsurface life, including below the seafloor in rocks and sediments) makes up a substantial portion of the planet and harbors vast amounts of microbial life. The Center for Dark Energy Biosphere Investigations (C-DEBI) specializes in the exploration of microbial life, geochemistry, and hydrology in the subsurface (NSF-funded Science Technology Center). Since C-DEBI was established (2010), the number of scientists with a primary focus on deep biosphere research has increased within the last decade as a direct result of efforts from C-DEBI. The objective of this white paper is to present the broad ideas of what the future of deep biosphere research may look like, from the perspective of early career researchers (graduate students, postdoctoral scholars, pre-tenure faculty).

\section{C-DEBI Early Career NextGen Workshop}

The primary objective of this professional development workshop was to promote the retention and success of the next generation of deep biosphere researchers. The workshop had two major components related to the ECR experience. The first component, held in several short sessions through the first 2-days, involved knowledge transfer between members of the C-DEBI leadership (Amend, Huber, and Wheat) and the workshop attendees. The C-DEBI leadership team provided presentations on 3 topics ("Effective proposal writing", "So you want some money to study the subsurface/deep sea?", and "Funding agency nuances") drafted from experience related to the topics and attendee submitted questions. Extensive Q\&A sessions occurred after each presentation to allow continued discussion for additional knowledge transfer to occur.

The second component sought to have the ECR attendees spend time considering the "big questions and challenges" that subseafloor and deep biosphere presently face while considering future research proposals that could be driven by C-DEBI ECRs. The 9 topics identified, along with recommendations from the group, are summarized below. This component utilized small group brainstorming activities, allowed attendees to self identify research commonalities, and provided an iterative framework structure to provide feedback from other attendees. The overall experience allowed the ECR attendees to identify both strengths and weaknesses in fledgling proposal ideas so that feasible and tractable ideas could be triaged away from ideas that were underdeveloped or beyond the limitations of current technologies.

\section{Discussion Topics: What are the biggest challenges facing deep biosphere research? \\ 1.1. Topic 1: Linking Bioinformatics to Physiological/Quantification Measurements}

Sequencing-based exploration of the environment continues to grow and provides a snapshot of microbial diversity that provides an avenue for data-driven hypothesis generation. However, these hypotheses require confirmation through various in situ and ex situ experiments to determine the direct impact on the environment and geochemical cycles.

1.1.1. Novel development of technology and sampling efforts that either (1) allow for the collection of samples to assess cellular processes and biomass for 
simultaneous sequencing and quantitative assays or (2) conduct experiments in situ.

1.1.2. It will be necessary to combine sequencing and experiment planning at the onset of research and not as an accessory to samples of opportunity (i.e., genome to phenome linkage).

1.1.3. This work will also require improved methodologies that can hone in on individual cellular processes and specific process rates (also see Topic 2).

\subsection{Topic 2: Low Biomass and Detection Limits}

Low cellular biomass and low cell-specific rates for energy acquisition (i.e., power requirements for maintenance) and growth (i.e., long doubling times) make studying active life in the deep biosphere especially challenging. Typical molecular tools for measuring activity, such as transcriptomes, require larger numbers of starting biomass than are found in typical deep biosphere samples. Further, the presence of DNA alone does not provide insight on whether a cell is dead, dormant or active, limiting our understanding of the physiological status of cells in the environment. Low cell-specific rates require unique ways to address how to study life that does not operate on human life scales and may not be directly observable in the laboratory. And while it may be possible in other environments to cultivate organisms of interest, the cultivation of microorganisms from low biomass requires extra care due to the higher susceptibility for contamination.

1.2.1. Molecular methods need to consider both contamination during the sampling process (i.e., drilling fluids) as well as from the processing of samples (i.e., kitome).

1.2.2. Require methods that evaluate the fine line between what is defined as 'alive' versus 'dead' in the deep biosphere (i.e., slow doubling times).

1.2.3. Emphasize approaches to obtain measurements on the scale of individual organisms rather than the bulk community to obtain cell-specific rates (also see Topic 1).

1.2.4. Acknowledge that targeted approaches are necessary to access the high proportion of uncultivated taxa found in the deep subsurface compared to surface environments may need specialized.

1.2.5. These driving questions need to be addressed:

1.2.5.1. Is current technology a barrier to going beyond detection limits?

1.2.5.2. Is more data and repeat measurements required to make sense of trends not currently understood?

\subsection{Topic 3: Non-model is the new model organisms}

The concepts of how life in the surface operates may not be applicable to the deep biosphere as these systems may have fundamentally different requirements for life. Similarly, many of the computational tools and approaches we use are derived from studies of model species or 
systems. This idea needs to be re-visited, and we must work towards de novo approaches or methods that evaluate the rules of life in the biosphere without bias. Similar considerations are required for experiments, such as cultivation, where expectations for microbes to behave, grow, and respond to their environments like their surface counterparts may prove unrealistic. If we apply the rules of the surface world to the subsurface, we cannot fully determine how the rules of life differ in the deep subsurface.

1.3.1. This requires the design of computational tools that consider the identification of novel elements within a query, as well as, extract meaning from data without representation in reference databases or similarity to model organisms. This may require designing schema that necessitates manual assessment.

1.3.2. The lack of representation of deep subsurface sequence data in our databases presents a challenge. The bias in representation inhibits new discoveries (i.e., subsurface specific traits and/or taxa). Additional sequencing data is needed from the vast diversity of subsurface habitats on Earth.

1.3.3. The rules of life (including how we define life) are different in the deep biosphere. Therefore we must invest in tools that are designed under the premise that we do not know the rules of life.

1.3.4. Novel cultivation methods are needed that go beyond classic cultivation approaches (i.e., axenic culture growing in exponential phase). Due to the slow doubling times in the deep subsurface, laboratory cultivation of deep subsurface microorganisms will often not be entirely reflective of their in situ physiological status.

\subsection{Topic 4: Large-Scale Global Models of the Subsurface}

The majority of information about subsurface biomass, metabolic activities and rates, are derived from only a few sites globally. This makes it inherently difficult to scale up from few sites to global scale models. Any concerted effort to accurately model the marine subsurface will require multiple new sampling efforts and explicit goals to expand these datasets. In addition to expanding the types of subsurface environments we have sampled from, there is a need to prioritize efforts that will ground truth existing models and address if our current observations reflect reality. It is clear that the 'microscale' processes that occur in the subsurface effect 'global scale' biogeochemical cycling (i.e., carbon sequestration). Substantial efforts are required to more fully understand how microscale processes are linked to local processes that are in turn linked to regional and global processes. Models need to account for the dynamic nature of communities that affect biogeochemical cycling in the subsurface and globally.

1.4.1. The subsurface is not heterogeneous and more sampling is required to obtain data on the diversity of sites globally.

1.4.2. Research is required to determine the sampling resolution necessary to constrain microscale to the regional/global scale processes.

1.4.3. It is necessary to move from empirical (data-driven) model to mechanistic (process-based) models. 
1.4.4. Emphasize models that will account for the temporal nature of the microbial communities that affect biogeochemical cycling, exploring subsurface life over time (i.e., 4D models).

\subsection{Topic 5: Investment in infrastructure}

The deep subsurface is logistically difficult and costly to access, investment in the infrastructure that facilitates this work is essential to the longevity of deep biosphere research. Infrastructure includes novel engineering solutions and the establishment of long-term field sites and observatories. There is the challenge to expand and maintain existing infrastructure (i.e., CORKs; Circulation Obviation Retrofit Kit), but also the opportunity to create community resources that can be accessed by scientists to sample these unique environments (e.g., remotely operated vehicle [ROV] and human-occupied vehicle [HOV] accessibility, but also hardware with specific uses like the Universal Fluid Obtainer [UFO]). The democratization of hardware infrastructure would expand the researchers that could explore questions in the subsurface and provide expedition platforms for others to participate in the research.

1.5.1. There are universal needs that require innovative engineering solutions: deep-sea plankton net, in situ sampling, or adaptive sampling, in situ experimentation.

1.5.2. Establishment of field sites and observatories that balance the need for frequent visits (i.e., Long Term Ecological Research programs) vs. new locations (see Topic 4).

1.5.3. Require innovative ways to retrofit existing CORK architecture to permit remote sampling and biological data collection. Prioritize future CORK infrastructure that would include these capabilities.

1.5.4. Maintain existing observatories and Research Coordination Networks (or equivalent efforts) and that ensure that collected data continues to be widely accessible.

\subsection{Topic 6: Increasing Diversity, Education and Inclusion efforts in the deep subsurface/Geosciences}

Diversity and Inclusion in science, technology, engineering, and mathematics (STEM) fields has received increasing attention in recent years, however the geosciences remain the least diverse of the STEM fields with little progress made in the past 40 years; the lack of progress of diversity in geosciences is a challenge for the future. Diversity, Education and Inclusion (DEI) efforts must continue and expand further to meet this challenge. A number of successful previous C-DEBI efforts were identified (e.g., Global Environmental Microbiology undergraduate programs, the C4 Research Experience for Undergraduates (REU) and CC-RISE programs, multilingual "15 Second Science" videos). Increased outreach for education would help increase the diversity of scientists carrying out deep biosphere research. The cost of working in the deep biosphere is usually high and inaccessible to many researchers globally which can create a 
colonialist approach and legacy in deep biosphere research. Deep biosphere work often involves fieldwork which can pose unique challenges for ensuring a safe and inclusive environment. This list is not exhaustive, and the required expertise to address DEI issues must be sought outside of the deep biosphere community to positively and productively co-create a more diverse and inclusive deep biosphere community. This topic cannot be adequately addressed in the scope of this whitepaper.

1.6.1. Fund sustained opportunities for undergraduate and graduate students that explicitly target black, indigenous, and people of color (BIPOC) and low income students as a method for increasing the number of diverse students to the STEM pipeline. Explicit mentorship programs are needed to retain diverse scholars at all levels.

1.6.2. Barriers to participating deep subsurface work must be identified at multiple levels (i.e., educational, national, and international) and efforts should be made to reduce or remove barriers, when possible. Proposal writers should be encouraged to consider colonialist approaches that impede access to the deep subsurface.

1.6.3. Removing barriers that are generally rooted in socioeconomic disparities (e.g., GRE standardized tests for graduate school, unpaid internships).

1.6.4. Continuing and enforcing an inclusive culture at sea and in the field (i.e., mandatory training for advisors, students, and staff). An essential part of these trainings is emphasizing how existing internalized cultures can lead to persistent microaggressions or dangers related to field work (e.g., countries where it is illegal to be homosexual). An inclusive culture at sea also involves envisioning how to make work accessible to differently abled persons.

1.6.5. Acknowledge that addressing DEI issues requires continued learning and self-reflection from individuals and institutions in the deep biosphere community and there is a need to incorporate outside expertise (i.e., DEI staff positions).

\section{Thoughts on the Subsurface Community of the Future}

2.1. Topic 1: Establishing a Reliable Funding Infrastructure for Deep Biosphere Research

Exploring the subsurface would require a large-scale investment for stakeholders as the community performs engineering and sampling feats similar to space exploration without the dedicated budget of agencies like NASA. Deep biosphere research is a relatively new field; to facilitate a continuous pipeline of personnel, and intellectual continuity, a stable, reliable and permanent funding infrastructure needs to be committed. Because the deep biosphere is unique in logistical challenges as well as rules of life, research in this field does not always fall within the domains of already existing research programs (e.g., ocean research does not mean the water that moves within the crust). Without a funding body that explicitly acknowledges the uniqueness of this field, deep biosphere research risks falling between the cracks of funding 
bodies. There needs to be an emphasis on retaining early career scientists in the field or there is a risk of losing progress without a continuation of the pipeline.

\subsection{Topic 2: Deep Biosphere as a Realm for Innovations}

The ECRs identified three areas that have the potential for direct societal impact and subsequently may function as engines of financial/logistical support for additional deep biosphere research. These areas are timely and provide opportunities for innovative collaborations with diverse disciplines. There is a need to quantify how microorganisms provide broad ecosystem services in deep subsurface environments. (1) Deep sea mining may become economically viable in the new future with initial tests for commercial manganese nodule mining efforts taking place in 2020. (2) The oceanic crust is being considered as a potential location to sequester atmospheric $\mathrm{CO}_{2}$ as carbonates as an effort to mitigate the climate crisis, as recently demonstrated in basalts in Iceland. (3) The deep sea biosphere harbors unknown microbial biologics that are essential for biotechnology approaches. Identification of novel secondary metabolites from the deep subsurface has the potential to generate revenue and provide a novel source of commercially viable antibiotics and antimicrobials. The vast amounts of novel microbial life forms and associated enzymes and biomolecules in the subsurface may be a source of potential biotechnological advances in medicine or other industries. The deep subsurface has significant potential for scientific advances of societal benefit in fields from medicine to climate change mitigation.

\subsection{Topic 3: A platform for multidisciplinary subsurface researchers}

The interdisciplinary community that has formed around C-DEBI, which should be maintained and used to establish new connections and collaborations. The C-DEBI community has been focused on core scientific themes within the deep biosphere, but has still tended to support smaller individual research plans over integrated approaches. It is common in the sciences to work in silos and compile or integrate across disciplines later. Looking forward, ECRs see opportunities to form innovative collaborations with even more sub-disciplines than have currently been established within the community. One way to ensure the interdisciplinary platform continues is to interact with researchers in other fields from the inception of an idea. The core themes of C-DEBI are interdisciplinary by nature; next steps of deep biosphere researchers likely include going beyond the original themes (i.e., linking biology to depositional history or paleoclimate work, linking sediment and niche seafloor habitats with abyssal and hadal regions of the water column).

\section{Conclusion}

The ECRs at the workshop view the discussed topics as markers along a road map towards a sustainable future in deep biosphere research. In addition to the challenges and opportunities outlined, a sustainable future includes intentional bridging of senior investigators with ECR, and requires integration of DEl efforts at every stage moving forward. DEI, multidisciplinary activities, and strategic investment are pillars of a sustainable future that must be prioritized. ECRs currently are DEI leaders, and are at the forefront to creating a more just and diverse scientific 
community. However without a platform and power in the long term (i.e., faculty positions, research positions) these ideas and role models will be lost. Finally, integrating ECRs through many channels will cultivate a culture of retention, support from senior scientists is vital. This includes involving ECRs in the planning stages of new long-term infrastructure or expeditions.

The C-DEBI NextGen 2019 workshop facilitated the discussion of the unifying challenges in the field, and the identification of priority objectives moving forward, from the perspective of ECRs. The deep biosphere remains a poorly understood and largely unexplored habitat with global scale implications. There is significant potential for continued innovation. The topics identified are broad in scope, but imperative for healthy scientific progress and the continuation of a lasting deep biosphere scientific community. 


\section{Executive summary of workshop activities}

C-DEBI NextGen was held November 9th-11th in Monterey, California and led by Sarah Hu, Jackie Goordial, and Ben Tully. In attendance were 17 Early Career Researchers (ECRs; including the 3 co-leads) ranging from postdocs to Assistant Professors that have either received previous funds from $\mathrm{C}-\mathrm{DEBI}$ or have collaborated on/contributed to C-DEBI sponsored research. In addition to ECRs, three senior scientists, Jan Amend, Geoff Wheat, and Julie Huber attended as invited guest speakers. The first day began with structured group brainstorming, where each participant contributed ideas and thoughts to address "What are the next big challenges for research in the deep biosphere?" These big ideas were re-visited often throughout the workshop; broadly they can be summarized as: (1.1) Linking bioinformatics to quantifiable measurements, (1.2) dealing with low biomass and detection limits, (1.3) viewing non-model systems as the new model, (1.4) integrating data for large-scale global models, (1.5) investing in infrastructure, (1.6) improving diversity, equity, and inclusion, (2.1) establishing a reliable funding resource, (2.2) viewing the deep biosphere as a realm of biotechnological innovations, and (2.3) increasing cross discipline collaborations. These ideas are compiled and summarized below to represent the broad ideas of the current early career 'cohort' associated with C-DEBI.

During each day of the workshop, invited guest speakers were asked to share their insight and be a part of a panel to address the nuances of obtaining funding to study the deep biosphere. Topics discussed included: how to request equipment from the National Deep Submergence Facility and shiptime, submitting proposals for drilling, how to gain access to already existing infrastructure (e.g., CORK subsurface observatories), and tips for writing proposals to NSF, NASA and other avenues such as foundations and private money. Ahead of the workshop, participants submitted questions and topics they wanted to learn more about and the invited speakers catered their talks and discussion to address these questions. The remainder of the workshop was spent brainstorming on big projects/proposals and presenting preliminary ideas to the group (and panelists) for immediate peer review. Projects ranged from planning preliminary lab experiments, outlining a review paper, to full proposals. The workshop concluded with a series of lightning talks where each group presented their project objectives, scientific hypotheses, and next steps. Post-workshop survey responses were positive, and participants greatly appreciated the panelists' talks. 